\title{
Assistência farmacêutica em unidades hospitalares em tempos de pandemia - uma revisão integrativa
}

\author{
Pharmaceutical assistance in hospital units in times of pandemic - an integrative review
}

\author{
Raylla Ketelly Bevenuto da Silva" ${ }^{\bullet}$, Marcio Trevisan ${ }^{\bullet}$
}

Faculdade de Palmas, Palmas, Tocantins, Brasil. *Autor para correspondência. E-mail: bevenutoraylla@gmail.com

\begin{abstract}
Resumo: Introdução: A pandemia da COVID-19 impôs aos farmacêuticos hospitalares a necessidade de planejarem e organizarem uma resposta aos desafios trazidos pela doença. Farmacêuticos exercem atividades fundamentais no contexto da pandemia, seja por meio do fornecimento dos medicamentos ou pela prestação dos serviços farmacêuticos. Gerenciar farmácias, hospitalares ou comunitárias, em tempos de SARS-CoV-2 é um novo desafio. A farmácia hospitalar vem enfrentando diversos desafios frente ao cenário da pandemia e, para continuar ofertando um serviço de qualidade à população, foi necessária a adoção de estratégias de enfrentamento e adaptação de suas atividades. Assim, esta pesquisa tem por objetivo evidenciar a importância do farmacêutico e das farmácias dentro das unidades hospitalares, considerando as especificidades da pandemia da COVID-19. Revisão: Trata-se de uma revisão bibliográfica de natureza exploratória, através de uma revisão integrativa. O levantamento de dados científicos e a sistematização das informações são oriundas de produções científicas publicadas de 2018 a 2021, no idioma português, indexadas na base de dados da BVS, LILACS e SCIELO. Discussão: Farmacêuticos hospitalares tiveram que reestruturar o serviço em resposta à COVID-19, considerando a necessidade de proteger as equipes, de forma que não haja comprometimento da atividade assistencial e de apoio à ação médica e, simultaneamente, da`rem uma resposta eficaz à nova realidade, não deixando de lado o componente de revisão e monitorização da utilização das tecnologias de saúde e a farmacovigilância ativa de fármacos usados no tratamento da COVID-19. Considerações Finais: Verificou-se que o conceito de farmácia hospitalar vai além de um setor de guarda de medicamentos sendo um um departamento que agrega valor a outros setores do hospital. Assim, os achados da pesquisa permitiram concluir, que farmacêutico, quando atuando em farmácias hospitalares, pode colaborar com a equipe multiprofissional em saúde acompanhando a utilização dos medicamentos e a evolução clínica dos pacientes visto que o uso off label de medicamentos é uma realidade atual que pode gerar sérios agravos a saúde dos indivíduos.
\end{abstract}

Palavras-chave: assistência farmacêutica, farmacêutico, farmácia hospitalar, pandemia.

\begin{abstract}
Introduction: The COVID-19 pandemic imposed on hospital pharmacists the need to plan and organize a response to the challenges brought about by the disease. Pharmacists exercise fundamental activities in the context of the pandemic, either through the supply of medicines or the provision of pharmaceutical services. Managing pharmacies, hospitals or community, in times of SARS-CoV-2 is a new challenge. The hospital pharmacy has been facing several challenges in the face of the pandemic scenario and, in order to continue offering a quality service to the population, it was necessary to adopt coping strategies and adapt their activities. Thus, this research aims to highlight the importance of pharmacists and pharmacies within hospital units, considering the specificities of the pandemic of COVID-19. Review: This is an exploratory bibliographic review, through an integrative review. The collection of scientific data and the systematization of information come from scientific productions published from 2018 to 2021, in Portuguese, indexed in the database of the BVS, LILACS and SCIELO. Discussion: Hospital pharmacists had to restructure the service in response to COVID-19, considering the need to protect the teams, so that there is no compromise in the care activity and support for medical action and, simultaneously, provide an effective response to the new reality, not neglecting the component of reviewing and monitoring the use of health technologies and active pharmacovigilance of drugs used in the treatment of COVID-19. Final Remarks: It was found that the concept of hospital pharmacy goes beyond a sector of custody of medicines, being a department that adds value to other sectors of the hospital. Thus, the research findings allowed us to conclude that pharmacists, when working in hospital pharmacies, can collaborate with the multiprofessional health team, monitoring the use of medicines and the clinical evolution of patients, since the off-label use of medicines is a current reality that can cause serious damage to the health of individuals.
\end{abstract}

Keywords: pharmaceutical assistance, pharmacist, hospital pharmacy, pandemic. 


\section{Introdução}

Em dezembro de 2019 o mundo começou a passar por uma intensa transformação, no âmbito da saúde mundial, quando na China foram identificados os primeiros casos de uma nova patologia que ataca as vias respiratórias de forma aguda, sendo essa provocada por um novo coronavírus. A Organização Mundial de Saúde, denominou a doença como COVID-19, vindo a declarar em março de 2020 que essa doença já havia se tornado uma pandemia global (Monteiro et al., 2020). Desde então, os profissionais farmacêuticos de todo o mundo têm buscado formas de responder, agir e se adaptar para manter a continuidade das atividades relacionadas a assistência farmacêutica e a qualidade dos serviços (Silva et al., 2020). Só no Brasil, até maio de 2021, já são mais de 14 milhões de casos confirmados e mais de 406 mil mortes (Brasil, 2021).

Os profissionais farmacêuticos que atuam na linha de frente contra a pandemia têm se tornado profissionais essenciais, uma vez que possuem total respaldo técnico para integrar as equipes de saúde, contribuindo com todo o seu conhecimento a respeito dos medicamentos, em especial, a doses, efeitos adversos e interações medicamentosas, dando suporte a outros profissionais e orientando os pacientes por meio da atenção farmacêutica. É de se esperar que após o término da pandemia, o profissional farmacêutico tenha mais visibilidade e responsabilidades visto que, neste momento, a importância da atenção farmacêutica é evidente a partir do que o mundo vivenciou com a COVID-19 (Rubert et al., 2021).

A pandemia da COVID-19 trouxe um cenário novo e desafiante a todo o sistema de saúde e à sociedade no geral, impondo aos farmacêuticos hospitalares a necessidade de planejamento e organização, visando dar uma resposta aos desafios trazidos pela COVID-19 (Farinha \& Rijo, 2020). Farmacêuticos exercem atividades essenciais no contexto da pandemia, tanto no nível ambulatorial quanto no hospitalar, seja por meio do fornecimento dos medicamentos ou pela prestação dos serviços farmacêuticos (Silva et al., 2020).

Até o momento, a divergência de protocolos para o tratamento da COVID-19 tem mostrado grande diversidade de condutas na prática clínica diária. O aumento preocupante e expressivo na venda de medicamentos sem prescrição profissional e a produção dinâmica de conhecimentos científicos, com vários resultados controversos, evidenciam a necessidade dos profissionais farmacêuticos incorporarem nas suas práticas os referenciais teóricos da farmacoepidemiologia e da avaliação de tecnologias em saúde, com vistas a assegurar um cuidado baseado em evidências (Martins \& Reis, 2020).

Aos farmacêuticos, como profissionais de saúde envolvidos no combate à pandemia por COVID-19, cabe a prestação e o funcionamento de um serviço essencial à população por meio das farmácias comunitárias ou hospitalares (Vaz et al., 2020). Gerenciar farmácias, sejam elas hospitalares ou comunitárias, em tempos de SARS-CoV-2 é um novo desafio (Santos \& Santos, 2020). A farmácia hospitalar vem enfrentando diversos desafios frente ao cenário da pandemia e, para continuar ofertando um serviço de qualidade à população, foi necessária a adoção de estratégias de enfrentamento e adaptação de suas atividades (Batista et al., 2020).

Além de atender às necessidades de medicamentos dos pacientes internados, as farmácias hospitalares também visam garantir o uso seguro e racional de medicamentos prescritos por profissionais médicos. É responsabilidade da farmácia hospitalar manter sob sua guarda os estoques desses produtos. Esses devem ser configurados de acordo com os ciclos de demandas e de reabastecimentos, devendo ser controladas as oscilações significativas e altos graus de indefinição, condições críticas frente a necessidade de assegurar medicamentos em disponibilidade na mesma igualdade de seu uso (Oliveira \& Garcia, 2020). A farmácia é um importante setor dentro das organizações hospitalares, estando diretamente ligada à assistência ao paciente, sendo responsável pelos medicamentos e materiais médico-hospitalares (Bezerril et al., 2020).

Medicamentos e insumos farmacêuticos são produtos que chegam a representar, financeiramente, até $75 \%$ do que se consome em um ambiente hospitalar (Oliveira \& Garcia, 2020). Por esse motivo, o gerenciamento eficaz dos insumos farmacêuticos nas unidades de farmácia dentro dos hospitais pode favorecer a redução de custos e maior eficiência nas atividades clínico-assistenciais realizadas dentro da instituição. Assim, um profissional farmacêutico capacitado evidencia sua importância dentro da farmácia hospitalar, uma vez que ele é o profissional que possui as ferramentas necessárias e conhecimento técnico para desenvolver todas as atividades da unidade tornando-se corresponsável pelos resultados da terapia realizada com os pacientes e o trabalho desenvolvido com os demais membros da equipe de saúde (Melo \& Oliveira, 2021).

Nessa perspectiva, esta pesquisa tem por objetivo evidenciar a importância do farmacêutico e das farmácias dentro das unidades hospitalares, considerando as especificidades da pandemia da COVID-19. Essa pesquisa levantou, através de uma revisão integrativa, conceitos e finalidades das farmácias hospitalares, funcionamento da assistência farmacêutica e serviços farmacêuticos nesses ambientes, como também explanou sobre a atuação do farmacêutico frente à COVID-19. 


\section{Revisão}

Esta pesquisa trata-se de uma revisão bibliográfica de natureza exploratória, através de uma revisão de literatura do tipo integrativa. O levantamento de dados científicos e a sistematização das informações são oriundas de produções científicas publicadas de 2018 a 2021, no idioma português, indexadas na base de dados da Biblioteca Virtual em Saúde (BVS), Literatura Latino Americana do Caribe em Ciências da Saúde (LILACS) e Scientific Electronic Library Online (SCIELO). Os descritores utilizados foram: assistência farmacêutica, farmacêutico, farmácia hospitalar e pandemia.

Medidas emergenciais para controle da disseminação do vírus SARS-CoV-2, exigiram adequações em diversas estruturas da sociedade, incluindo os serviços de saúde. Dentre estes, encontra-se a assistência farmacêutica, na qual a dispensação de medicamentos, por ser considerado um serviço essencial para controle dos sintomas da COVID-19, não pode ser descontinuada (Zocratto et al., 2020). Com a pandemia da COVID19, a assistência farmacêutica, na sua prática diária, sofreu modificações significativas para se adaptar às novas demandas da população e dos serviços de saúde. As ações têm sido desenvolvidas conforme as demandas de cada região e sua disponibilidade de recursos financeiros e humanos (Martins \& Reis, 2020).

Serviços farmacêuticos são um conjunto de atividades contínuas e integradas, tendo o medicamento como elemento essencial no sistema de saúde, a fim de atender às necessidades de saúde das comunidades, famílias e indivíduos, contribuindo para prevenção de doenças e para proteção, promoção e recuperação da saúde (Tritany \& Tritany, 2020).

Assistência farmacêutica quando comparada com serviços farmacêuticos, possui um sentido mais abrangente (Soares et al., 2020). Dessa forma, a assistência farmacêutica é conceituada pela legislação como um conjunto de ações voltadas à promoção, proteção e recuperação da saúde, tanto individual quanto coletivo, tendo o medicamento como insumo essencial e visando o acesso e ao seu uso racional. Este conjunto envolve a pesquisa, o desenvolvimento e a produção de medicamentos e insumos, bem como a sua seleção, programação, aquisição, distribuição, dispensação, garantia da qualidade dos produtos e serviços, acompanhamento e avaliação de sua utilização, na perspectiva da obtenção de resultados concretos e da melhoria da qualidade de vida da população (Abreu et al., 2020).

A evolução da assistência farmacêutica tem um papel fundamental e importante na reestruturação da profissão farmacêutica, nesse sentido, a farmácia hospitalar deve ser administrada, exclusivamente, por um profissional farmacêutico, que deve estar ligado a direção do hospital e integrada com as demais unidades de assistência ao paciente (Travassos et al., 2018).

A farmácia hospitalar é a unidade clínica de assistência técnica e administrativa, sob a responsabilidade do profissional farmacêutico, é integrada, obedece uma hierarquia e de forma funcional ligadas às atividades hospitalares, buscando melhor qualidade de assistência, através do uso seguro e racional de medicamentos e produtos para saúde, ajustando sua aplicação à saúde individual e também coletiva. A Sociedade Brasileira de Farmácia Hospitalar define o serviço da Farmácia hospitalar como um componente sistêmico da organização hospitalar, caracterizando-a como uma referência clínica, administrativa e econômica (Trajano \& Comarella, 2019).

As farmácias têm por finalidade dispensar medicamentos sob a prescrição médica, na quantidade e especificações solicitadas, no prazo requisitado, oferecendo segurança e favorecendo o uso racional dos medicamentos e correlatos à população. Com o objetivo de contribuir para a assistência de saúde, as farmácias hospitalares, nos últimos anos, têm evoluído e se organizado, e com o foco no paciente e suas necessidades, têm em seu domínio o medicamento como instrumento, desse modo, sua prática e organização podem prevenir que erros relacionados a dispensação ocorram e causem danos à saúde do paciente (Oliveira \& Garcia, 2020).

A Portaria $n^{\circ} 4.283$, publicada em 2010, pelo Ministério da Saúde objetivou desenvolver e traçar diretrizes para consolidar a farmácia clínica no Brasil. Esse instrumento salienta que o principal foco da farmácia hospitalar é garantir o abastecimento, dispensação, controle, acesso, rastreabilidade e uso racional de medicamentos e produtos hospitalares. Além disso, é também de responsabilidade da farmácia hospitalar otimizar a relação entre custo, benefício e riscos de tecnologias, e principalmente desenvolver ações da assistência farmacêutica (Fernandes, 2019).

O conceito atual de farmácia hospitalar é moldado na existência de duas grandes áreas da assistência farmacêutica: atividades de suporte e atividades clínicas. A primeira envolve todas as atividades de carácter mais mecanizado, por exemplo farmacotecnia e distribuição, que funcionam em suporte às atividades clínicas. O uso apropriado dos fármacos exige a sua dispensação segura, rigorosa e eficiente, pelo que a automatização de processos é fundamental, apesar desta não ser o componente mais crucial para o seu uso apropriado. Já 
nas atividades clínicas, a maior contribuição do farmacêutico resulta da redistribuição das tarefas distributivas e deslocação para a tomada de decisões no campo da farmacoterapia e sua monitorização. A deslocação dos farmacêuticos hospitalares para as atividades clínicas tem assentado no pressuposto da manutenção de serviços de apoio eficientes garantindo a confiabilidade e qualidade dos processos e permitindo o desenvolvimento gradual de atividades clínicas no sentido de proporcionar maior valor ao paciente, baseados numa prática pela excelência (Farinha \& Rijo, 2020).

O farmacêutico, no âmbito hospitalar, atua em toda a cadeia medicamentosa, desde a aquisição dos medicamentos até a dispensação segura ao paciente. Esse profissional deve atuar também na seleção e padronização de medicamentos, na elaboração de diretrizes clínicas e protocolos terapêuticos, na promoção de informações sobre medicamentos e outros produtos para saúde assim como na participação de estudos e custo e efetividade (Batista et al., 2020).

$\mathrm{Na}$ linha de frente, o farmacêutico encontra-se habilitado para orientar e promover o uso racional dos medicamentos, realizar testes rápidos para a COVID-19, acompanhar os casos mais simples, notificar os casos suspeitos e encaminhar ao atendimento médico ou hospitalar para detecção do diagnóstico mais preciso (Rubert et al., 2021).

Ainda que a COVID-19 traga incerteza e se preveja um futuro difícil, os serviços farmacêuticos desempenham um papel crucial para atenuar as consequências adversas da pandemia. $O$ farmacêutico hospitalar é parte integrante de uma equipe multidisciplinar em saúde que trabalha em conjunto na prestação dos melhores cuidados de saúde para o doente (Pereira et al., 2020).

\section{Discussão}

A existência de padrões de qualidade, regras claras para logística de insumos, medicamentos, e reciclagem, com atualizações periódicas, dos profissionais da saúde, colabora para aliviar o fardo e o colapso do sistema de saúde (Novaes, 2021).

Na visão de Farinha \& Rijo (2020), farmacêuticos hospitalares tiveram que reestruturar o serviço em resposta à COVID-19, pois existe uma necessidade latente de proteger as equipes, organizando-se para não haver um comprometimento da atividade assistencial e de apoio à ação médica e, simultaneamente, darem uma resposta eficaz face à nova realidade, não deixando de lado o componente de revisão e monitorização da utilização das tecnologias de saúde e a farmacovigilância ativa de fármacos usados no tratamento da COVID-19.

Rubert et al. (2021) evidenciam que a Assistência Farmacêutica durante a pandemia da COVID-19 é de extrema importância, uma vez que o farmacêutico exerce papel fundamental no controle da transmissão da doença e na atenção às necessidades da população, de forma a promover o uso racional de medicamentos durante a crise. Já Batista et al. (2020) evidenciaram que a falta de um tratamento específico revelou o papel essencial do farmacêutico no monitoramento de novos protocolos clínicos estabelecidos na comunidade científica com o uso off-label de algumas drogas, para garantia do uso racional de medicamentos e segurança do paciente

Nessa perspectiva, Batista et al., (2020) dizem que é função do farmacêutico hospitalar, não apenas atuar nos limites da farmácia, mas acompanhar a farmacoterapia do paciente através da validação de prescrições e participação em visitas multiprofissionais para intervir, quando necessário, no ajuste de doses de medicamentos, frequência de uso, adequação da via de administração, adequação da forma farmacêutica, duração da terapia, entre outros aspectos relevantes, visando garantir a segurança e a eficácia da terapia medicamentosa do paciente, resultando em uma recuperação mais rápida com consequente diminuição do tempo de internação.

Mais do que a dispensação de medicamentos, Melo \& Oliveira (2021) e Bezerril et al. (2020) destacam que as farmácias hospitalares também têm como função a organização dos produtos, aquisição, logística, sistemática de distribuição de medicamentos e correlatos. Os farmacêuticos hospitalares também devem participar das equipes de controle de infecção hospitalar, gerencia os resíduos, dá suporte nutricional e quimioterápico, atua no controle de qualidade, farmácia clínica, informação, farmacovigilância, ensaios clínicos, radiofarmácia e cuidados farmacêuticos, como também atua na educação em saúde.

Além das atribuições no âmbito da farmácia hospitalar, um grande desafio do farmacêutico nas unidades hospitalares é a farmácia clínica, que para Gribner (2019) a aplicação da farmácia clínica mostra-se como uma prática na qual os farmacêuticos prestam cuidados aos pacientes com o objetivo de maximizar o tratamento empregado. Como recurso pode-se optar por diferentes abordagens que envolvem a revisão da prescrição 
clínica, entrevistas e consultas, aconselhamentos e acompanhamento aos internados, discursão de casos clínicos com outros profissionais da equipe, entre outros. Para a autora, a participação do farmacêutico na equipe multiprofissional em um ambiente hospitalar vem crescendo expressivamente com o passar dos anos, o que contribui com o fortalecimento e consolidação da atenção farmacêutica e sua prática clínica.

Por fim, Fernandes (2019) conclui que o serviço hospitalar farmacêutico é um setor com autonomia técnica e científica, sendo a responsabilidade obrigatoriamente assegurada por um farmacêutico hospitalar, e estabelece uma estrutura importante aos cuidados de saúde dispensada no meio hospitalar. E também responsável pela orientação dos pacientes internados e ambulatoriais, visando sempre a eficácia terapêutica, racionalização dos custos, ensino e pesquisa, colaborando assim para um vasto campo de aprimoramento profissional.

\section{Considerações finais}

Atualmente, espera-se que o serviço de farmácia contribua diretamente para os resultados da assistência prestada aos pacientes e não atue apenas nas atividades de provisão de produtos e serviços. Diante do cenário de pandemia por COVID-19, o farmacêutico hospitalar pode contribuir fundamentalmente para o acesso, a qualidade, a efetividade e a segurança do medicamento, através da assistência farmacêutica. Tais contribuições estão relacionadas ao seu conhecimento profissional, e se materializam por intermédio da análise adequada das situações, da formulação de estratégias, da atuação multiprofissional e interdisciplinar, e do estabelecimento de estratégias inovadoras para garantir a segurança e o uso racional de medicamentos.

Os conceitos extraídos da pesquisa puderam evidenciar que a farmácia hospitalar é uma unidade de caráter clínico e assistencial, dotada de capacidade administrativa e gerencial, vindo a ser um dos setores mais importantes do hospital. Este setor é responsável pela provisão segura e racional de medicamentos e de materiais médico-hospitalares.

Verificou-se que o conceito de farmácia hospitalar vai além de um setor de guarda de medicamentos. É um departamento que agrega valor a outros setores do hospital, tornando necessário a adequada assistência aos pacientes. Assim, os achados da pesquisa permitiram concluir, que o farmacêutico, quando atuando em farmácias hospitalares, pode colaborar com a equipe multiprofissional em saúde, acompanhando a utilização dos medicamentos e a evolução clínica dos pacientes visto que o uso off label de medicamentos é uma realidade atual que pode gerar sérios agravos a saúde dos indivíduos que já estão internados em estado grave e situação crítica.

\section{Referências}

Abreu, R. D. S., Miranda, K. S., Simões, A. B., Vieira, G. D., \& Sousa, O. V. .2020. Assistência farmacêutica em unidades básicas de saúde: um foco no serviço farmacêutico. Brazilian Journal of health Review, Curitiba, 3(4), 9797-9911.

Batista, L. M., Oliveira, H. A. G., Vasconcelos, A. S., Fernandes, D. B. S., \& Cavalcanti, U. D. N. T. 2020. Mudanças da atuação multiprofissional em pacientes com COVID-19 em unidades de terapia intensiva. Health Residencies Journal, Brasília, 1(7), 1-20.

Bezerril, M. S., Silva, R. T. D., Santos, F. J. S., \& Salvador, P. 2020. Estratégias de gestão da qualidade e gestão de materiais de uma farmácia hospitalar: revisão integrativa da literatura. Revista de Administração Hospitalar e Inovação em Saúde, Belo Horizonte, 17(1), 97-110.

Brasil, Ministério da Saúde. 2021. Covid-19: Brasil tem 406,4 mil mortes e 14,7 milhões de casos. Disponível em: https://www.gov.br/saude/pt-br

Farinha, R., \& Rijo, J. 2020. Os Farmacêuticos Hospitalares Durante a Pandemia COVID-19. Revista Portuguesa de Farmacoterapia, Alcabideche - PT, 12(1-2), 9-19.

Fernandes, L. L. 2019. A importância do farmacêutico hospitalar juntamente com a equipe multidisciplinar na Unidade de Terapia Intensiva (UTI). Revista FAROL, Rolim de Moura, 8 (8), 5-21.

Gribner, C. 2019. Consolidação do farmacêutico na prática clínica. Revista Visão Acadêmica, Curitiba, 20(2), 48-58.

Martins, M. A., \& Reis, A. M. 2020. O farmacêutico no enfrentamento da COVID-19 no Brasil: onde estamos? Revista Brasileira de Farmácia Hospitalar Serviços de Saúde, 11(3), 1-2. 
Melo, E. L.; \& Oliveira, L. S. 2021. Farmácia hospitalar e o papel do farmacêutico no âmbito da assistência farmacêutica. Revista JRG de Estudos Acadêmicos, 4(8), 287-299.

Monteiro, F. F. C., Araújo, I. G., Santos, R. O., Nascimento, R. M., Loureiro, L. M. A., Pessoa, E. T. F. P., \& Morais, A. C. L. N. 2020. O papel da assistência farmacêutica no tratamento de pacientes com COVID-19 em hospital de referência no município de Fortaleza. Revista de Casos e Consultoria, 11(1), 1-7.

Novaes, H. M. 2021. Acreditação hospitalar e o enfrentamento ao covid-19. Revista de Administração em Saúde, 21(82), 1-8.

Oliveira, S. M. M., \& Garcia, M. A. T. 2020. Gestão de processos de dispensação de medicamentos em unidades hospitalares. Revista Científica Eletrônica de Ciências Aplicadas da FAIT, 2, 1-9.

Pereira, M. T., Oliveira, R., Baptista, M., Cardoso, J., Vilaverde, R., Cabeças, T., Sá, F., Pinto, B., Ventim, A. M., Ribeiro, G., Franco, S., Cunha, M., Gouveia, I. M., \& Galvão, M. 2020. Os Serviços Farmacêuticos CUF Infante Santo no Combate à Pandemia COVID-19: Dias Difíceis, Dedicação Incondicional. Gazeta Médica, Lisboa, Portugal, 7(2), 156-162.

Rubert, C., Deuschle, R. A. N., \& Deuschle, V. C. K. N. 2021. Assistência farmacêutica durante a pandemia da COVID-19: revisão da literatura. Revista Interdisciplinar de Ensino, Pesquisa e Extensão, 8(1), 255268.

Santos, D., \& Santos, J. N. 2020. As Farmácias Comunitárias na Pandemia COVID-19: Alianças Estratégicas em Contexto de Incerteza. Revista Portuguesa de Farmacoterapia, Alcabideche, 12(1-2), 53-55.

Silva, M. J. S., Moraes, E. L., Livinalli, A., Fonseca, C. L., Torriani, M. S., Gonçalves, P. N., Risch, R. O., \& Pereira, N. M. L. 2020. Nota Técnica da Sociedade Brasileira de Farmacêuticos em Oncologia sobre Assistência Farmacêutica em Oncologia frente à Pandemia de Covid-19. Revista Brasileira de Cancerologia, 66, 1-7.

Soares, L. S. S., Brito, E. S., \& Galato, D. 2020. Percepções de atores sociais sobre Assistência Farmacêutica na atenção primária: a lacuna do cuidado farmacêutico. Revista Saúde em Debate, 44(125), 411-426.

Trajano, L.C.N., \& Comarella, L. 2019. Gestão Farmacêutica na farmácia hospitalar: aumento da qualidade e segurança ao paciente e racionalização de recursos. Revista da Faculdade de Ensino Superior de Floriano, FAESF, 3(2), 4-8.

Travassos, D. L., Santos, S. R. L., Santos, N. D., \& Gardenghi, G. 2018. Atuação do farmacêutico hospitalar na redução de custos com medicamentos. Revista Eletrônica Saúde e Ciência, Goiânia, 8(2), 54-63.

Tritany, R. F., \& Tritany, E. F. 2020. Serviços Farmacêuticos no Enfrentamento à COVID-19: Uma Revisão Integrativa da Literatura. Revista Saúde em Redes, Porto Alegre, 6(2), 7-24.

Vaz, I., Serrão, C., Teixeira, A., Castro, L., \& Duarte, I. 2020. O Impacto da COVID-19 nos Farmacêuticos Portugueses: Fadiga e Exaustão Emocional. Revista Portuguesa De Farmacoterapia, Alcabideche, Portugal, 12 (3), 9-10.

Zocratto, K. B. F., Pereira, G. G., Oliveira, P., \& Santos, A. S. 2020. Impactos da pandemia da COVID-19 na dispensação de medicamentos pela assistência farmacêutica da regional de Pirapora. Revista Gestão \& Saúde, Brasília, 11 (3), 357-370.

\section{Minicurrículo}

Raylla Ketelly Bevenuto da Silva. Bacharelanda em Farmácia, cursando o último ano pela Faculdade de Palmas - FAPAL. Atua em uma farmácia hospitalar em uma unidade de pronto atendimento na cidade de Palmas- TO.

Márcio Trevisan. Doutor em Ciências do Ambiente pela Universidade Federal do Tocantins. Mestre em Ciências Biológicas - Bioquímica Toxicológica - pela Universidade Federal de Santa Maria. Especialista em Gestão de Vigilância Sanitária e Especialista em Regulação em Saúde no SUS pela Fundação de Ensino e Pesquisa Sírio Libanês. Graduado em Farmácia e Bioquímica pela Universidade Federal de Santa Maria. Atuou como farmacêutico na Secretaria Municipal de Saúde de Palmas. Atua como Analista de Saúde/Inspetor Sanitário no Núcleo de Educação em Vigilância Sanitária da Vigilância Sanitária de Palmas. Atua como 
docente de graduação acadêmica desde 2006. Atualmente é membro do Núcleo Estadual de Segurança do Paciente do Estado do Tocantins.

Como citar: Silva, R.K.B., \& Trevisan, M. 2021. Assistência farmacêutica em unidades hospitalares em tempos de pandemia - uma revisão integrativa. Pubsaúde, 7, a180. DOI: https://dx.doi.org/10.31533/pubsaude7.a180

Recebido: 4 mai. 2021.

Revisado e aceito: 19 mai. 2021.

Conflito de interesse: os autores declaram, em relação aos produtos e companhias descritos nesse artigo, não ter interesses associativos, comerciais, de propriedade ou financeiros que representem conflito de interesse.

Licenciamento: Este artigo é publicado na modalidade Acesso Aberto sob a licença Creative Commons Atribuição 4.0 (CC-BY 4.0). 